\title{
Arquitectura Contemporánea en la Periferia, La Biblioteca España y La Casa de la Lluvia: Un análisis estructural de contenido.
}

Oscar Mauricio Pérez

Maestro en Historia y Teoría del arte la Arquitectura y la Ciudad, Universidad Nacional de Colombia, Arquitecto de la Universidad Católica de Colombia. En la actualidad se desempeña como docente investigador de la Facultad de Arquitectura de la Universidad La Gran Colombia. Oscar.perez@ugc.edu.co ID 0000-0002-5902-5101

\section{Fabián Andrés Llano}

Doctorando en Ciencias Humanas del Patrimonio y la Cultura Universidad de Girona (España) Magister en Investigación social interdisciplinaria de la Universidad Distrital Francisco José de Caldas, Licenciado en Ciencias Sociales de la misma universidad. En la actualidad se desempeña como docente investigador de la Facultad de Arquitectura de la Universidad La Gran Colombia fabian.1lano@ugc.edu.co ID 0000-0003-2181-3476

Mireya Barón Pulido

Docente-investigadora del departamento de comunicación. Politécnico GrancolombianoBogota/Colombia. mg. lingüista- seminario Andrés bello/instituto Caro y Cuervo. langue francaise -paris iv-sorbonne. docente área de lenguajes. Investigadora en el campo del periodismo, comunicación mediática. investigaciones publicadas: 1: "la ciudad desde el periodismo local universitario" (redicom, conlacom-bolivia 2007. 2: "sintaxis digital en ciberperiodismo universitario-Bogotá" (agenda Alaic 2008-México, tecnológico de monterrey) 


\section{Resumen:}

El presente artículo analiza dos formas de construcción social y cultural del territorio en contextos de periferia. Los proyectos analizados son La Biblioteca España (2007-2015) y La Casa de La Lluvia (2013- Actualidad), el primero diseñado por El Equipo Mazzanti, en el marco de las políticas del Urbanismo Social que caracterizaron a la ciudad de Medellín en el periodo de 2004 al 2007 y el segundo construido por Arquitectura Expandida, un colectivo que opera desde el año 2010 teniendo su epicentro en la ciudad de Bogotá.

\section{Design/methodology/approach}

Para esta investigación realizó un análisis estructural de contenido a partir la información recolectada en fuentes como entrevistas, artículos y video documentales. Del análisis se extrajeron los modelos culturales presentes en la concepción de construcción del hábitat presentes en cada uno de los proyectos de arquitectura

\section{Findings}

Entre los hallazgos se evidencian dos modelos contemporáneos de ciudad y se destaca la manera en que las tendencias locales de acción urbana tienen presencia en el contexto local convirtiéndose en propuestas replicables. Se examinó papel del proyecto de arquitectura como un dispositivo de activación social, cultural y también mediático y su papel en la construcción de la imagen de ciudad.

Originality/value

La originalidad de esta investigación se encuentra en la aplicación de un análisis sociológico a dos proyectos arquitectónicos, se busca evidenciar dos modelos culturales de construcción del hábitat aparentemente incompatibles en cuanto a su concepción y su ejecución, pero común fin común: lograr el bienestar social de las comunidades de los contextos periférico.

Palabras clave: City branding, Participación social, Urbanismo social, Arquitectura Contemporánea 


\section{Introducción}

En la actualidad considerar la construcción de espacios colectivos y de la vivienda popular urbana implica pensar en nuevas relaciones, nuevas tipologías funcionales y nuevos valores en relación con una arquitectura comprometida (Waisman, 1985). Desde este punto de vista, la arquitectura requiere asumir los problemas más sobresalientes de la vida social, como lo son la segregación socio-espacial y la marginalidad urbana, que bajo un claro compromiso con la sociedad y la cultura fortalecen la idea de una profesión que potencia la comprensión de las dinámicas presentes en la ciudad contemporánea.

Para lograrlo, el arquitecto requiere incorporar a su praxis, una crítica constante a la institucionalización de la arquitectura como saber profesional y una lectura atenta del contexto para fortalecer su función social. El arquitecto contemporáneo necesita articular sus dinámicas profesionales con un constante ejercicio de reconocimiento del contexto mediante trabajo en campo, que junto con las posibilidades de la interdisciplinaridad pueda reconocer las diferentes problemáticas sociales sobre el territorio y la ciudad. De este modo, una mayor comprensión de las estructuras conceptuales de la práctica, el conjunto de objetos, problemas y estrategias de actuación en el territorio serán elecciones que refuerzan el futuro de su actuar.

La discusión sobre el papel de la arquitectura invita a descubrir una serie de lenguajes que hacen parte del hecho arquitectónico como construcción no solo espacial, sino también territorial. De acuerdo con Saldarriaga (1996) el objeto de estudio de la arquitectura no parte de construcciones teóricas y metodológicas propias de un lenguaje estético y del acto proyectivo, más bien surge como un hecho construido social y culturalmente, la arquitectura se ha nutrido de las diferentes visiones de las Ciencias Sociales y Humanas. En este sentido, el cuestionamiento principal de esta investigación fue descubrir ¿Cuál es el modelo cultural presente los proyectos: La biblioteca España y la Casa de La Lluvia para un territorio excluido? 


\section{Marco teórico:}

Para la siguiente investigación se optó por un marco teórico capaz de desarticular las representaciones asociadas al hábitat bajo el urbanismo tradicional y las posibilidades de la sociología para comprender no solo las valoraciones espaciales sino la relación directa entre unos espacios ocupados con su valoración social. De acuerdo con Llano la ciudad como una elaboración en el espacio, posee la cualidad de generar múltiples valoraciones dentro de diversos grupos sociales; capacidad que se ve reforzada si se tiene en cuenta el papel que desempeñan diferentes instancias, como la política, comunicación y las relaciones de fuerza que se presentan en su relación con la arquitectura (2014)

Así estas valoraciones espaciales y sociales de la ciudad requieren de un tratamiento espacial en cuanto al abordaje de territorios excluidos y estigmatizados. A partir de esta perspectiva teórica se examinó por medio de un análisis crítico la prácticas territoriales del Urbanismo Social y la Arquitectura Colectiva, tomando como caso de estudio La Biblioteca España en Medellín y La Casa de la Lluvia en Bogotá, utilizando para ello información bibliográfica, audiovisual y testimonios exponiendo los alcances que van desde lo global hasta lo local y desde lo legal hasta lo legítimo, evidenciando el manejo que se le da a temas como la imagen, el diseño participativo, la relación con el contexto histórico, social y cultural en la intervención barrial de la periferia.

\section{De las conceptualizaciones del hábitat a la identificación del espacio excluido y segregado}

La reducción de la idea de hábitat a solo alojarse y detentar un espacio, excluye las relaciones antropológicas y sociológicas en la medida en que el habitar es un hecho antropológico. La habitación, la mansión, el hecho de fijarse al suelo (o de desprenderse de él) el hecho de arraigarse (o de desarraigarse) el hecho de vivir aquí o allá (y por consiguiente el hecho de partir, de ir a otra parte) estos hechos y este conjunto de hechos son inherentes al ser humano (Lefebvre, 1975, p154). El término hábitat proviene de las ciencias biológicas y de la antropología, y quizás la idea del hábitat de los pueblos primitivos, considerado como un medio ambiente integrado entre si y a la vida del grupo humano, sirvió de base a la traslación del concepto para caracterizar el espacio vital del hombre moderno, para el cual la vecindad, el barrio, la ciudad, deberían ser extensiones coherentes del espacio (Waisman 1985, p41). 
En oposición a esta idea, el uso generalizado del concepto de hábitat sin inscribir a las diferentes iniciativas sociales relacionadas con la construcción colectiva termina por excluir las prácticas sociales y las estructuraciones espaciales propias de un territorio.

\section{Los nuevos escenarios de la arquitectura: la exclusión y la periferia de la ciudad}

Es más, esta significación social del espacio es desprovista muchas veces de reflexión colectiva y lo que resulta mucho más problemático en este sentido, son las intencionalidades sociales veladas o naturalizadas en la producción del espacio. Para Bourdieu (2001) en las relaciones espaciales se pueden observar las objetivaciones del espacio social. En esta complejidad se hace evidente una lucha constante entre diferentes posiciones por la clasificación social, producto de las estructuraciones económicas sociales y culturales, que, sometidas a la fuerza de la historia, evidencian desigualdades como fenómenos naturales y los expone como productos terminados e inalterables. Los efectos de las estructuras económicas y sociales sobre el espacio proceden bajo procesos de constantes acatamientos de las valoraciones y devaluaciones sociales y espaciales. Así en la lucha territorial se dan las diferentes disposiciones y tomas de posición de los agentes sociales en un espacio de posibilidades denominado por Bourdieu espacio social.

En efecto, como afirma Wacquant (2006), las apreciaciones que desconocen las configuraciones históricas y sociales ocultan las diferencias sociales y económicas en las dinámicas urbanas, amparadas en políticas de invisibilización de la pobreza, políticas que posibilitan la estigmatización de espacios que tienden a la involución económica y al deterioro social. En este sentido, las valoraciones espaciales presentes en los barrios marginales sometidos a la segregación socio-espacial, frente al reciente fenómeno del urbanismo social y de la arquitectura colectiva, que implican concebir las intervenciones en barrios periféricos y así mismo la instrumentalización de la arquitectura para cambiar la imagen de la ciudad.

\section{Lo metodológico: el análisis estructural de contenido}

A partir de esta perspectiva teórica se examinó por medio de un análisis crítico la prácticas territoriales del Urbanismo Social y la Arquitectura Colectiva, tomando como referentes la Biblioteca España en Medellín y La Casa de la Lluvia en Bogotá, utilizando contenidos como 
información bibliográfica, audiovisual y entrevistas para exponer los alcances que van desde lo local hasta lo global hasta lo local y desde lo legal hasta lo legítimo exponiendo el manejo que se le da a temas como la imagen urbana, el diseño participativo, y la relación con el contexto histórico, social y cultural en la intervención barrial.

En términos metodológicos se procedió bajo el análisis estructural de contenido, que por lo general se aplica a materiales reducidos bajo la pregunta sobre ¿cuál es el sistema de sentido y el modelo cultural manifestado por este material? El análisis se interesa en los contenidos que se desprenden de diversos materiales concretos como entrevistas, relatos, testimonios e imágenes. Habría que aclarar que estos materiales no son concebidos como discursos y por tanto no se corresponde con el análisis de los mismos. (Hiernaux, 2008) Este análisis parte del supuesto epistemológico asociado a una estructuración de los contenidos en el pensamiento de los sujetos, que valga decir, proceden bajo sistemas de percepciones. En realidad el contenido expresa estos sistemas dentro del texto que relaciona el contenido con unos sentidos. Lo que persigue el análisis estructural de contenido son las persistencias culturales que se pueden expresar en modelos culturales. Para esto se hace necesario una perspectiva teórica ligada a la sociología de la cultura donde se comprende el modelo cultural como géneros diferentes de sistemas de sentido socialmente producidos (Hiernaux, 2008)

De acuerdo con Hiernaux, (2008), estos géneros se expresan como guías que orientan el comportamiento y funcionan como principios organizadores de la percepción y el comportamiento. De allí que se proceda metodológicamente a conocer el sistema de sentido para hacer previsible los conceptos en cuanto a un conocimiento interiorizado, socialmente producido, reproducido y transformado. Ahora bien, estas prácticas, comportamientos y haceres pueden ser restituidos bajo formas discursivas diversas que se pueden esquematizar desde planteamientos greimacianos sobre la base de dos mecanismos argumentativos: la disyunción y la asociación. Por el primero se entiende, la contra definición, la distinción al interior de un mismo género al identificar ciertas representaciones que se despliegan unas en función de otras, por ejemplo interior en función del exterior. Desde la segunda operación, se procede bajo conjunciones donde los elementos restituidos en la disyunción se asocian con diferentes atributos o calificaciones. En la investigación propuesta se determinó en primer lugar el estatuto teórico de los materiales mediante entrevistas en profundidad, se partió del 
presupuesto metodológico que los mejores materiales para el análisis estructural de contenido, son aquellos donde las personas se expresan libremente y sin imposiciones de tiempo y control de variables. (Hiernaux, 2008) De allí, los datos emergentes fueron dispuestos por los investigadores como unidades de análisis bajo un inventario del material observado. Luego de determinar las unidades de análisis se pasó a identificar las disyunciones elementales de estas manifestaciones culturales, con el propósito de demarcarse de lo no eran para estructurar las parejas de contra definiciones. La tercera operación metodológica tuvo que ver con la verificación de las asociaciones entre unidades y términos de las parejas de contra definiciones y por último se extrajo los grafos que esbozaron los modelos culturales asociados a la imagen de la ciudad y a las formas de hacer ciudad.

Los resultados del análisis implican los montajes culturales bajo cuatro operaciones principales. La extracción de las bases empíricas, la emergencia del modelo cultural por disyunciones y asociaciones, la visibilización de grafos sobre el modelo cultural para hacerlo generalizable y las formas de abstracción privilegiadas por los investigadores que fue forma de abanico.

\section{Resultados}

El análisis de contenido realizado a propósito de los casos propuestos para este análisis, muestra como la biblioteca España, puede señalarse como el proyecto eje del modelo Medellín y del urbanismo social. Desde la forma el edificio es concebido como un icono formado por tres cuarzos negros posadas en uno de los cerros más altos del Valle de Aburra, en el barrio Santo Domingo Sabio (Mazzanti, 2013) ${ }^{1}$. Este proyecto busca fortalecer una imagen y hace que lo visual sea lo fundamental (Gonzales 2015). Sin embargo, dentro del análisis de contenido, aunque intenta convertirse en una imagen que se debate entre la existencia barrial y la aceptación de los parques biblioteca, resulta en una contraposición entre una imagen de orden metropolitano versus orden barrial. (Diagrama 1).

\footnotetext{
${ }^{1}$ El edificio fue reconocido con múltiples galardones, entre los que se destaca, el mejor proyecto arquitectónico en la VI Bienal de Arquitectura Iberoamericana (Rodríguez, 2008), a nivel artístico, es el único proyecto colombiano cuyas maquetas reposan en la colección del MoMA Museo de Arte Moderno de Nueva York (Mazzanti, 2012).
} 


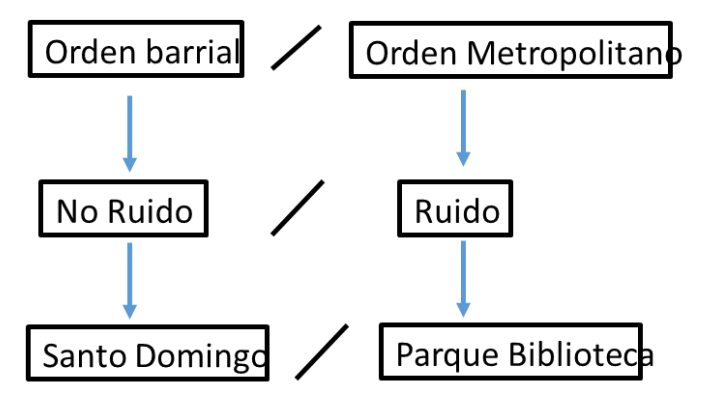

Imagen de Ciudad

Panto Doming $/$ Parque Bibliotec

Procesos de Urbanización

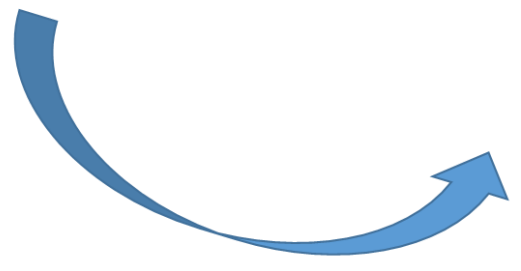

\section{Autoconstrucción}

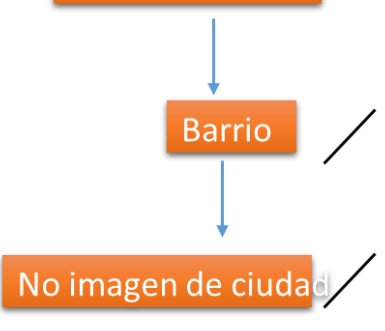

\section{Construcción}

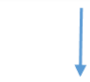

Estado

Imagen de Ciudad

Figura 1, muestra como en el caso de Medellín se privilegia al orden metropolitano frente al orden barrial al generar mayor presencia mediática (ruido) traducido a una imagen de ciudad producida por la construcción estatal .La imagen de ciudad se produce bajo la creencia de que la autoconstrucción no genera una imagen de ciudad.

Aparece la contraposición social de este proyecto de índole metropolitano frente a los procesos de autoconstrucción. En este sentido, la imagen contrapuesta de un proyecto como la biblioteca España que buscaba convertirse en un éxito, en los sentidos y los significados entró en contraste con las lógicas de concepción informal del barrio periférico como proceso de urbanización alejado del Estado, esto provocó un interés internacional sobre los procesos urbanísticos de la ciudad logrando transmitir la idea de la superación de la violencia por medio de la arquitectura. La arquitectura como salvadora de la violencia.
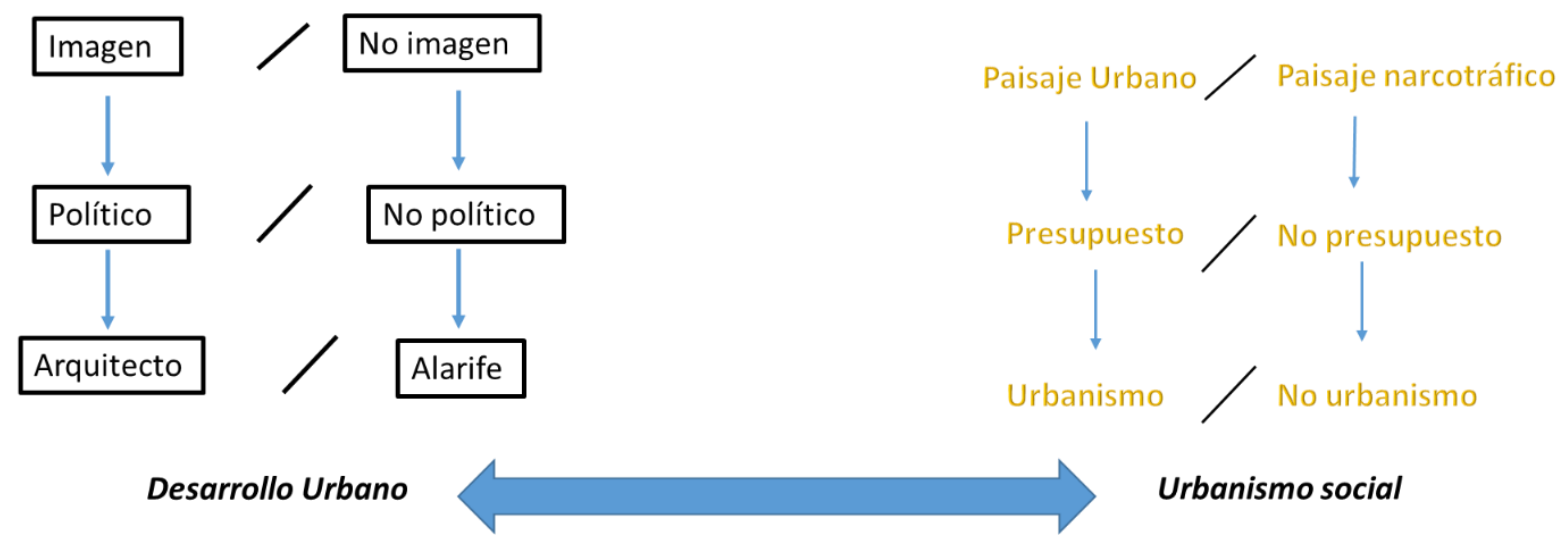

Urbanismo social 
Figura 2 El arquitecto, a diferencia del alarife cumple un papel político, suple de imagen a lo que no tiene imagen. El urbanismo aparece como un ente ordenador, necesario y su inversión es necesaria para producir un paisaje urbano.

Esto se observa en la implantación del proyecto no se tuvieron en cuanto a las condiciones específicas del lugar; el edificio obstruye la visual de edificios dedicados a funciones colectivas, en lugar de considerarse a la hora de diseñar la propuesta: "A espaldas de las bibliotecas, quedan la placa polideportiva el centro de salud, la iglesia, el proyecto nos tapó la vista hacia el occidente, así mismo nos quitó caminos” (Rocha, 2014).

Otro asunto relevante se da en la definición de participación ciudadana en el desarrollo del proyecto, la cual fue casi nula, los talleres de participación posteriormente al diseño de la biblioteca, en palabras de uno de los pobladores, Rosalba Cardona: "La administración nunca le ha preguntado a la comunidad que podía ser lo que la comunidad necesitaba" (Rocha, 2014). De esta forma, el urbanismo de este tipo de intervenciones cae en una óptica generalizada, excluyendo las estructuras espaciales existentes en el lugar, así como sus prácticas sociales (Ver Figura 3). El proyecto no tuvo en cuenta un diálogo previo al diseño, y negó de esta forma la posibilidad de un proceso consensuado que valorara las dinámicas presentes en el sector, así como las estructuras físicas como lo fueron viviendas de los pobladores que fueron expropiadas llevando a algunos pobladores a desalojar sus casas. Según el propio alcalde Sergio Fajardo, al inicio de la construcción de la biblioteca se dió una fuerte oposición de parte de la comunidad, (Escallon, 2013) quienes no estaban de acuerdo en aspectos como la localización o el uso del mismo, existían "Vacíos en cuanto inversión estatal a unos cuantos metros de la biblioteca" como precisa Manuel Maheca, (Rocha, 2014). 

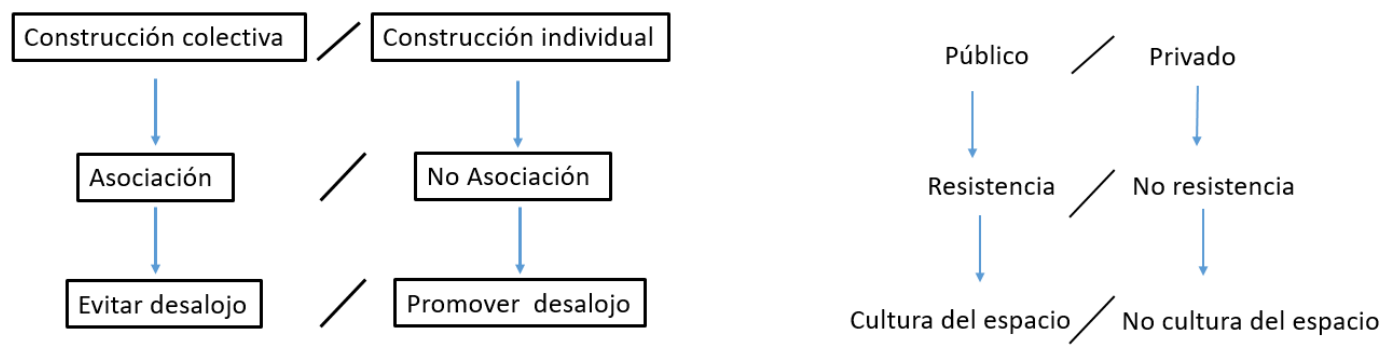

Modelo espacial de construcción del territorio

Percepciones sobre la construcción del territorio

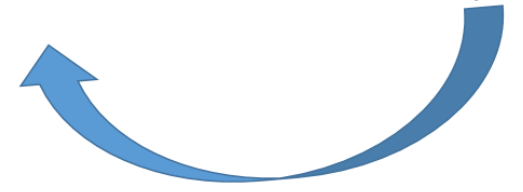

Figura 3. En lugar de promover la construcción colectiva, la construcción individual evita la asociación y de esta forma promueve el desalojo, los tiempos de los proyectos son distintos a los tiempos de la comunidad. El escenario de lo público evita también la valoración de las resistencias barriales como una parte fundamental de los procesos, esto produce un proceso de homogenización que no reconoce la cultura espacial presente en el barrio.

Como consecuencia de lo anterior y desde el análisis de contenido, la imagen que se proyectó fue una imagen contrapuesta entre el paisaje del narcotráfico y el paisaje urbano contemporáneo, donde el primero se asocia a la falta de presupuesto de innovaciones tecnológicas y escasa presencia del urbanismo en el espacio público, mientras que la imagen contemporánea asume la presencia de un paisaje urbano bajo el predominio del urbanismo social y el impacto directo del espacio público.

Es una imagen contrapuesta mediada por lo estético, entre una imagen negativa de la ciudad, y una imagen positiva. Desde la primera hay una ausencia de reconocimiento de la ciudad asociado a la persistencia de la violencia y a la escasa participación política en temas de simbolización de la ciudad, desde la imagen positiva se muestra una ciudad abierta a los negocios, a los constructores y una participación abierta a los políticos en obras para la ciudad. (Ver figura 4 )Cabe resaltar que dentro de las medidas de normalización del barrio una de las estrategias que acompañó la intervención de la Biblioteca España fue el incremento de la fuerza pública, con la policía de turismo (Arasanz, 2010) se buscó crear un 
escenario amable para el turista, logrando con ello la invisibilización de las condiciones del barrio y teniendo un mayor control del sector. Como precisa Jorge Corrales, "La paz como indica uno de los habitantes del sector, nos llegó de reflejo cuando llegó el metrocable, digo de reflejo porque es sabido que Las autoridades no vinieron a cuidarnos a nosotros habitantes de santo domingo, sino a cuidar estas infraestructuras que cuestan bastantes millones de pesos, pero bienvenida la paz de reflejo”. (Rocha, 2014)

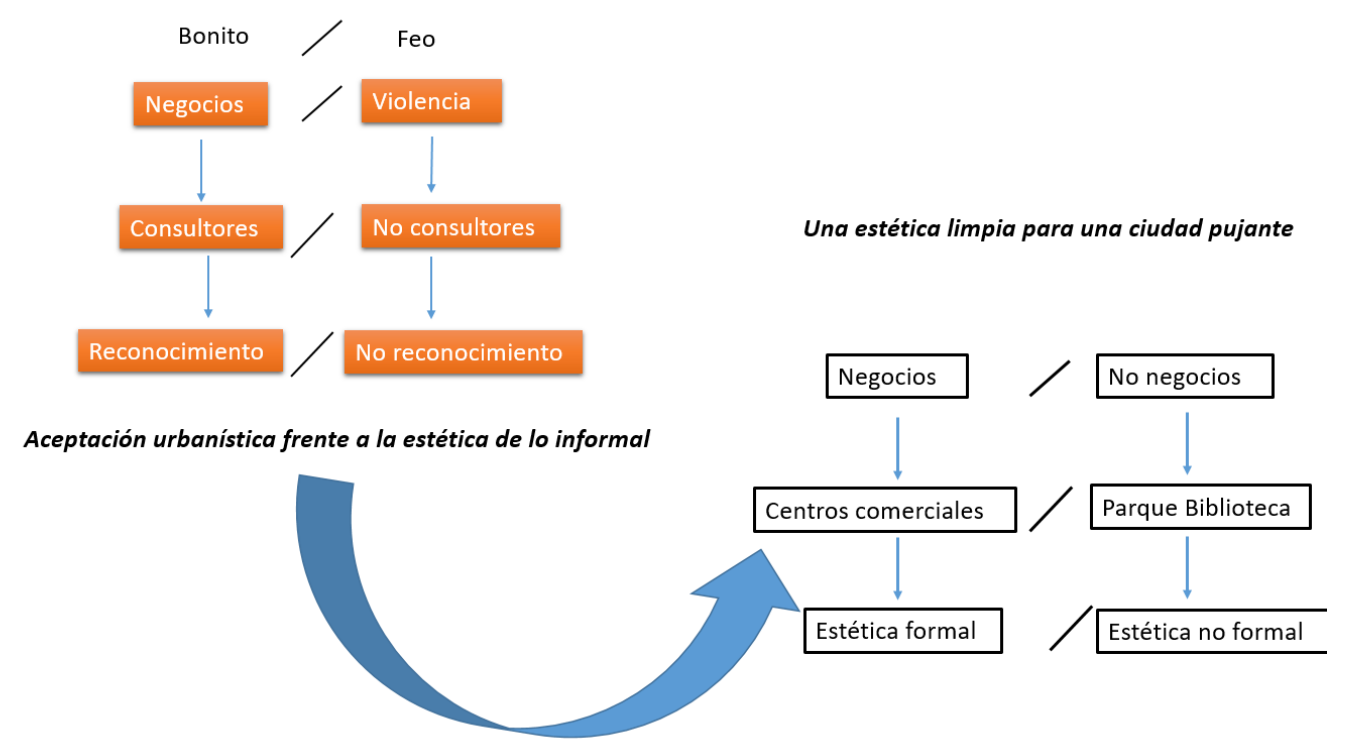

Figura 4 En lugar de reconocer las expresiones y las materialidades propias del contexto, se opta por una imagen y unificada a través de una estética reconocible, esto no reconoce la estética informal

De esta forma, el edificio crea una imagen de seguridad y bienestar transmisible tanto para el turista como para el lector internacional. Este proyecto privilegia la imagen estética al contenido social. No se tuvieron en cuenta las preexistencias del lugar, ni su cultura, ni su historia. Como solución a la segregación espacial en los barrios periféricos, termina produciéndose otro tipo de segregación. Al no volver partícipe a las comunidades de las decisiones tales como la localización, los programas, los diseños e incluso las formas de construcción de los edificios, se está vetando una posibilidad de actuación colectiva sobre el territorio. Lo importante de la presencia de las bibliotecas públicas en la ciudad de Medellín no se relaciona sólo con el impacto urbanístico, paisajístico o arquitectónico sino además y, 
aún más importante, con el significado, la valoración y la utilidad social que tienen las bibliotecas públicas y su proyecto cultural, educativo, político y económico en las comunidades que atiende Bornacelly, et al (2014)

Actualmente el edificio se encuentra en estado de abandono, de manera irónica, un proyecto cuyo eje central fue la producción de imagen, tuvo que cerrar sus servicios debido al desprendimiento de su fachada. En el año 2015 un informe estructural producido por la Universidad Nacional de Colombia indicó que, debido a la deficiencia de las especificaciones técnicas, la instalación de la fachada no contó con los anclajes adecuados para soportar las cargas y esto causo que las piezas de piedra se desprendieran ocasionando su progresivo cierre.

Representantes de la comunidad como Manuel Mahecha, han reconocido que la biblioteca proyecta una imagen hacia la ciudad y hacia el mundo (Rocha, 2014). Teóricos como Duque han expuesto el concepto de corresponsabilidad, a través de la cual se atribuye a la gente un papel protagónico como promotores y actores principales de la transformación de sus sectores, bien sea con su implicación en la definición de las intervenciones o con su apropiación en un marco de civismo, convivencia y control social, que permitan la sostenibilidad de las actuaciones. (Duque 2014). Son numerosos los teóricos, urbanistas arquitectos que coinciden en este punto, es urgente en este tipo de intervenciones procesos de concertación y de diseño participativo que fomenten un verdadero diálogo entre el programa institucional y el programa barrial logrando de esta forma que la integración en los procesos de ordenamiento territorial sean lo suficientemente agudos o visionarios. (Rocha, 2014).

\section{La Casa de La Lluvia de ideas}

El proyecto insignia del colectivo Arquitectura Expandida ubicado en la localidad de San Cristóbal en Bogotá se ha convierte en un instrumento para la conservación barrial y la lucha territorial siendo parte de un proceso de consolidación del barrio La Cecilia. Aquí la imagen del proyecto se vuelve un símbolo que busca exaltar las lógicas de construcción del territorio, tales como el autoconstrucción, la autogestión, la recursividad en los materiales e incluso la fragilidad de los mismos (Figura 5). Algunos dispositivos son implementados de forma con materiales flexibles y livianos, que incluso podrían ser objeto de vandalismo. Si con el tiempo 
son respetados, o incluso protegidos por los distintos actores de la calle, habrá llegado el momento de que sea consolidado, como intervención definitiva. (Valencia, 2015).

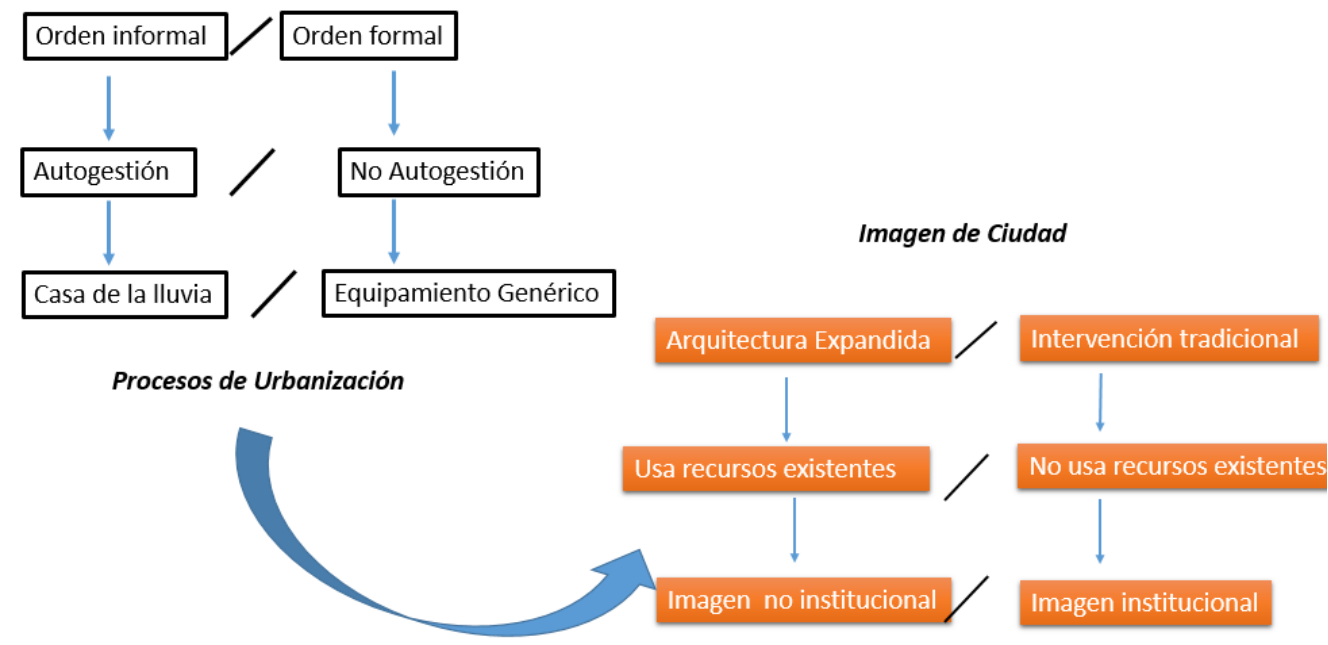

Figura 5 La Casa de la Lluvia se construye utilizando a las lógicas presentes en la informalidad, el autoconstrucción, esto lleva a un equipamiento que se piensa desde las necesidades y los medios de la población.

A diferencia de las intervenciones tradicionales, la arquitectura colectiva utiliza los recursos existentes y los conocimientos propios de la población y la mezcla con técnicas cercanas y económicas como el uso de la guadua, esto genera una imagen no institucional para el equipamiento.

Aun cuando el proyecto genera unos beneficios para la comunidad y ha funcionado como un catalizador de otros proyectos sociales, hay que resaltar que es parte de una serie de procesos del barrio y allí encuentra sus propias lógicas, frente a la colonización barrial, el empoderamiento esto diferencia el diseño participativo del diseño colectivo (Figura 6). Lo importante para nosotros es que al final haya estructuras sociales, quizás eso parte de que el problema no es arquitectónico, es social, educativo, de autogestión, participación política, etc. Cuando uno siente que estos componentes existen, ya hay un germen de eso, si creemos que la arquitectura, la autogestión en la construcción de espacios culturales y de encuentro es una herramienta más dentro de un proceso. (Lopez, entrevista personal, 24 de agosto 2016) 


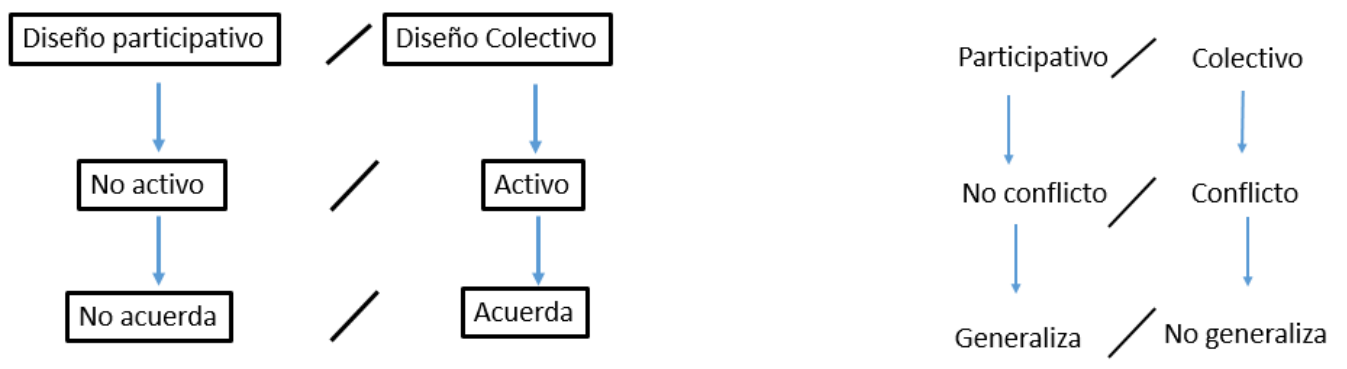

Modelo espacial de construcción del territorio

Percepciones sobre la construcción del territorio

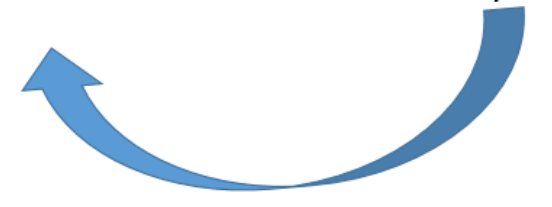

Figura 6: El diseño participativo se ha utilizado como un instrumento para legitimar proyectos públicos, sin embargo, la participación se limita a la consulta dejando a la comunidad en un papel pasivo frente a las decisiones de los proyectos. El diseño colectivo a diferencia busca evidenciar y resalar los conflictos presentes en el territorio y través de estas tensiones que se construye. Cada territorio cuenta con unas problemáticas particulares que no se pueden generalizar.

A nivel de escala el edificio a penas cuenta con $60 \mathrm{~m} 2$, a lo lejos, de día a penas y destaca entre la aglomeración de viviendas, el proyecto busca ser un medio de inclusión de la comunidad, tanto en el sentido de recursos sociales, como de recursos materiales y culturales, la construcción misma del edificio se convierte en un evento social, un carnaval que busca llamar la atención de la comunidad, y encontrar un apoyo cada vez mayor por parte de los vecinos del barrio, al equipo de trabajo también se integran personas de otras disciplinas y otros medios tales como cine, periodismo, arte, sociología interesados en ayudar en estos procesos (Figura 7).Lo importante para nosotros es que al final haya estructuras sociales, quizás eso parte de que el problema no es arquitectónico, es social, educativo, de autogestión, participación política, etc. Cuando uno siente que estos componentes existen, ya hay un germen de eso, si creemos que la arquitectura, la autogestión en la construcción de espacios culturales y de encuentro es una herramienta más dentro de un proceso. (Lopez, 2016) 


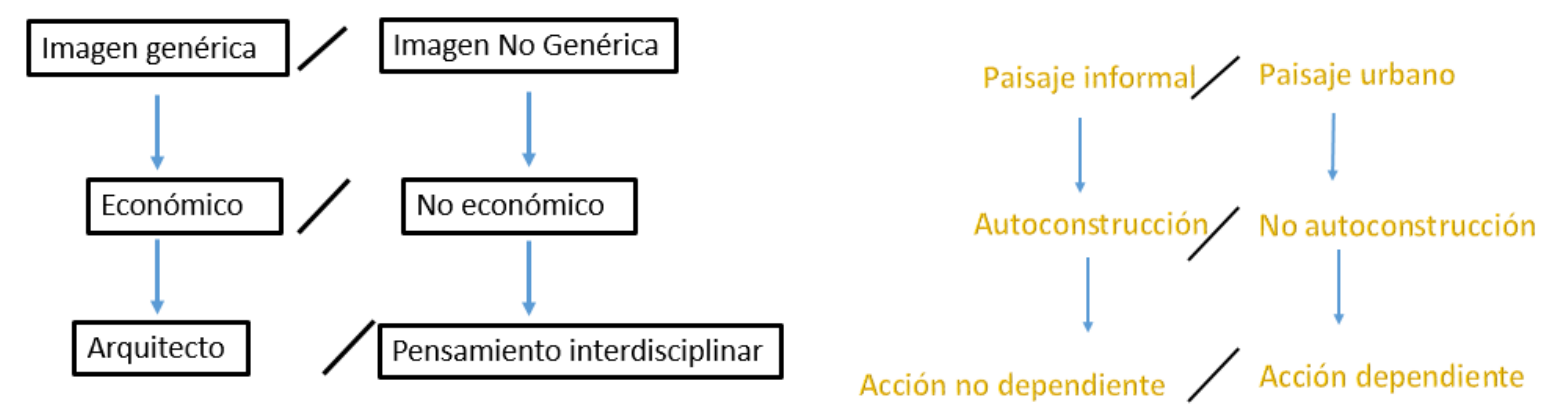

Desarrollo Urbano

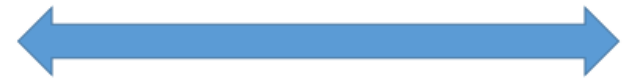

Arquitectura colectiva

Figura 7 : Parece existir una creencia por la cual la presencia de un pensamiento interdisciplinar alarga los tiempos de un proyecto arquitectónico volviéndolo no económico. A mediano y largo plazo esto es falso, ya que las propuestas genéricas pueden resultar insostenibles si no tienen en cuenta los procesos sociales presentes en un territorio. La mayor fortaleza del paisaje informal radica en que el autoconstrucción no depende del estado, el paisaje urbano tradicional delega su construcción y mantenimiento a otros.

En lugar de imponer un programa externo, con unas formas diseñadas en una oficina de arquitectura, arquitectura expandida realiza prácticas de diseño participativo como medio de trabajo con los habitantes del barrio, esto desde luego no implica que dejen de lado la cuestión de la imagen. Los proyectos del colectivo buscan generar un impacto tanto al interior de la comunidad como afuera y este se nota en aspectos como la concepción del nombre de la intervención, o la fabricación de los logotipos de cada proyecto que se conciben de forma colectiva (López, 2016). Arquitectura expandida utiliza medios informáticos para visibilizar sus procesos. Cada intervención es documentada y organizada, a través de mapas, obras cinematográficos, escritos, diagramas y guías de montaje, las cuales funcionan además como una herramienta pedagógica, este material esta disponibles en su sitio web, esto con el fin de promover la replicabilidad de estas iniciativas (Arquitectura Expandida, 2014). El diseño, gráfico y arquitectónico se vuelve un instrumento que orienta y construye la imagen colectiva (Figura 8). 


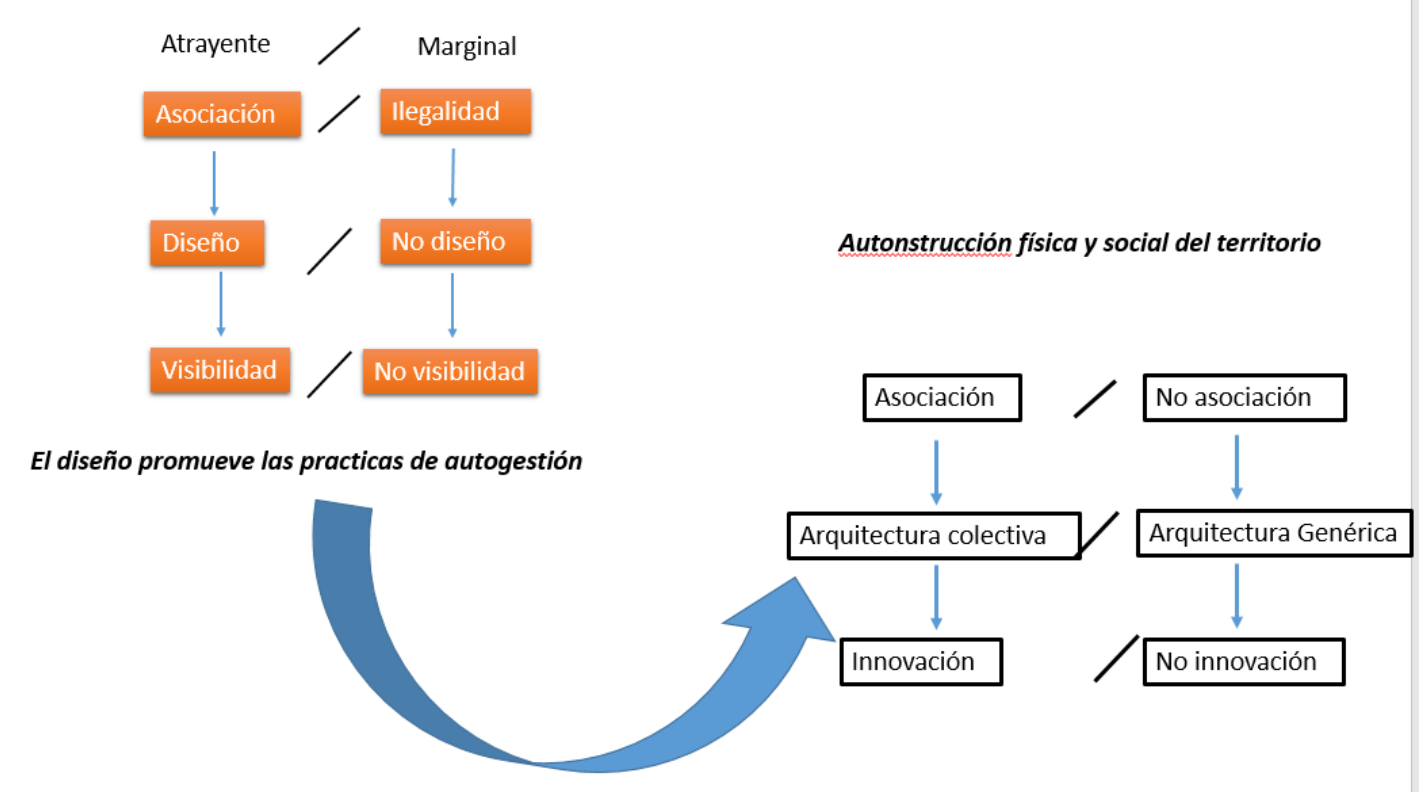

Figura 8: En lugar de ver la periferia como un entono marginal, ilegal e invisible, las prácticas de Arquitectura Expandida buscan darle visibilidad a los procesos sociales y materiales de la informalidad la clave de esto es la asociación y el diseño. Las prácticas de arquitectura expandida resultan innovadoras al juntar las lógicas presentes en la periferia a las prácticas de un estudio de arquitectura y un colectivo artístico, esto genera una imagen y unos procesos que difieren de la práctica tradicional de la arquitectura.

Así mismo, con estas acciones se busca generar una mayor pertenencia al territorio. Más que un marco legal para las propuestas, se busca una legitimidad que surja de las intenciones de los ciudadanos (Orjuela, 2017). A diferencia de las propuestas del urbanismo social no se busca generar seguridad a partir del incremento de la fuerza pública, sino más bien lograr mayor confianza a la hora de construir y habitar lo público. La arquitectura colectiva funciona como un dispositivo de empoderamiento ciudadano (Pinzón, 2011), dando lugar a un desarrollo horizontal del que los ciudadanos son directamente promotores, los proyectos surgen de procesos de resistencia (Valencia, 2015).

La casa de la Lluvia se mantiene hoy en día, después de 5 años de su ejecución, pertenece a la red de bibliotecas barriales de la ciudad y presta diferentes servicios a la comunidad, entre los que se destacan servir de espacio a talleres y eventos comunitarios, ensayos de grupos musicales, danza entre otros. El proyecto ha cambiado desde su inauguración incluyendo jardinería interior, y un mural sobre uno de los costados del mismo, y aun cuando los materiales no están hechos para una larga duración se han conservado en los últimos años. El colectivo arquitectura expandida ha conseguido participar en la construcción de proyectos 
similares tales como El Trebol, y la primera sala de cine de Ciudad Bolivar, Potocine. El colectivo también ha incursionado en instalaciones artísticas como la recientemente presentada para el Royal Academy of Arts y procesos de investigación a través de los cuales hacen públicas sus estrategias colectivas.

\section{Conclusiones}

Es cierto que la Biblioteca España en principio presentó beneficios para la comunidad del Barrio Santo Domingo al dotarlo con una infraestructura e incentivar ampliamente factores como el turismo y el comercio del barrio, también incrementó los índices de lectura y participación en eventos culturales y talleres comunitarios. Sin embargo es necesario evaluar de una manera crítica la pertinencia este tipo de mega proyectos en contextos periféricos. Para empezar, Este proyecto fue concebido desde una oficina de arquitectura, sin tener en cuenta las preexistencias en el barrio Santo Domingo Sabio y así mismo, no se consultó a la comunidad sobre temas como la localización del proyecto eligiendo uno de los lugares menos aconsejables a nivel sísmico y teniendo más en cuenta la visibilidad de la misma. La razón de esto fue la implantación de un modelo cultural que buscaba producir edificios iconos que mostraran al mundo el cambio social que estaba ocurriendo en la ciudad de Medellín. La Biblioteca junto con varias acciones urbanísticas en barrios periféricos de la ciudad generó una imagen de cambio a nivel internacional y dio pie para cambiar la marca de violencia de la ciudad, por la marca de la innovación.

El principal problema del modelo cultural que representa la Biblioteca España radica en su escala, al orden metropolitano sobrepuesto al orden barrial, esto ocasiona un desarrollo insostenible, al concebir un proyecto para mejorar la imagen de la ciudad y no para solucionar las necesidades puntuales del barrio se desperdicia una oportunidad para incentivar los procesos sociales existentes y ser más coherente con las lógicas de construcción del territorio. El orden metropolitano suprime las lógicas de construcción del barrio así como la participación de la comunidad en temas como la gestión, el diseño y la construcción del mismo. El futuro actual de la biblioteca Santo Domingo es incierto, el deterioro del edificio ocasionó que desde el 2015 la Biblioteca cerrara sus puertas y hasta la fecha no se ve ha concebido un plan de reconstrucción del mismo. Allí hay una nueva oportunidad para hacer las cosas de otro modo. 
En arquitectura la escala es un atributo relativo, la dimensión de un edificio no es sinónimo de profundidad. El modelo de ciudad planteado por La Casa de la Lluvia presenta una intervención urbana mucho menor, es casi 100 veces la Biblioteca España y además cuesta casi 250 veces menos. Sin embargo la mayor diferencia entre estos dos proyectos y la razón de la sostenibilidad de La Casa de La Lluvia no radica en su costo económico sino en su valor social, el hecho de que la iniciativa de construcción surja desde la comunidad es un ejemplo de empoderamiento ciudadano y de procesos de autoconstrucción política y social del territorio. El proyecto es coherente con la tradición y las lógicas presentes en el barrio periférico en cuanto a procesos de gestión, diseño y construcción. La arquitectura colectiva se presenta como un medio para ampliar las relaciones sociales concibiendo la construcción

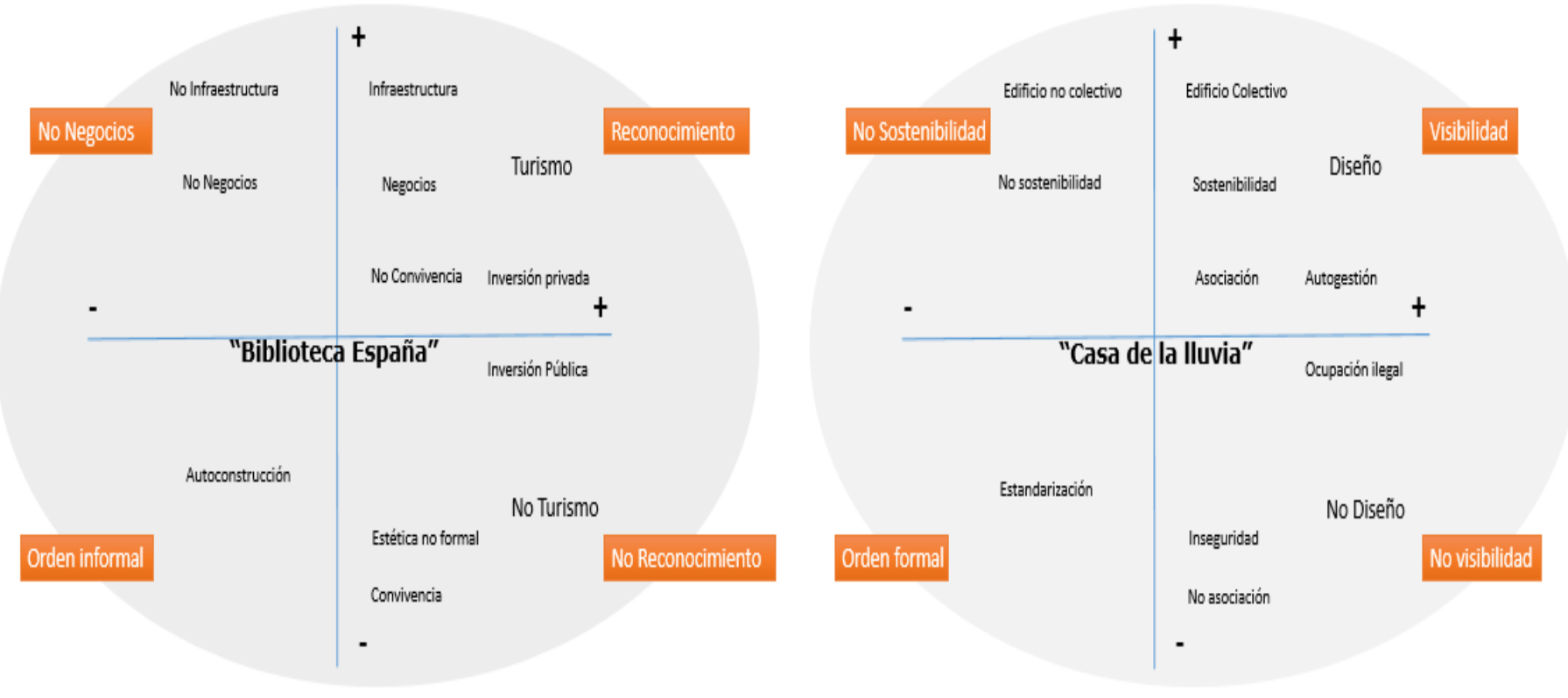

del edificio como la extensión del espacio vital de sus habitantes, y tiene como fin la producción de un hábitat participativo y propositivo. (Ver figura 9)

Figura 9: Expone los modelos de ciudad a partir del análisis estructural de contenido, mientras en la biblioteca España se persigue el reconocimiento, mediante la superación de la violencia mediante la arquitectura, en la Casa de la Lluvia, se busca visibilizar los procesos de construcción presentes en el barrio. Mientras que en el primer modelo existe una oposición entre las dinámicas del 
barrio y la imagen de turismo, en la segunda se da una oposición entre la imagen del barrio y las prácticas genéricas que se dan en las propuestas urbanas

¿Es posible que el Estado actúe como un facilitador de estos procesos colectivos suministrando el apoyo necesario e incluya en su desarrollo los tiempos y los procesos barriales?

Desde la experiencia de Arquitectura Expandida y su proceso investigativo, en el contexto actual, es poco probable escalar las intervenciones de arquitectura colectiva para convertirlas en una política pública. Esto principalmente por una estructura jurídica y administrativa rígida que limita las alternativas en las dinámicas de participación, pasando por limitaciones como la contratación y licitación que pueden desvirtuar los procesos colectivos (López, 2016). A esto hay que sumarle la voluntad política del gobierno de turno, así como la incompatibilidad de los tiempos burocráticos y los procesos barriales. Esto deja como alternativa para los proyectos de arquitectura colectiva moverse entre las fisuras jurídicas presentes norma, reivindicando la autoconstrucción del territorio como un medio para ejercer una desobediencia civil consciente y política en permanente construcción y la reconstrucción de sus derechos urbanos.

\section{Referencias}

Bourdieu, P. (1999) "Efectos de lugar" in Bourdieu, P. (Ed): La miseria del mundo, Fondo de Cultura Económica. México

Escallòn, J. (2003) Maravillas de Colombia: Biblioteca España - Discovery en la Escuela. Available at: http://discoveryenlaescuela.com/guias/maravillas-de-colombia-biblioteca-espana/ (Accessed: 1 August 2018).

García, J,H. (2013) "Slum tourism, city branding and social urbanism: the case of Medellin, Colombia", Journal of Place Management and Development, Vol. 6 Issue: 1, pp.43-51 
Hiernaux, D. (2008), “Análisis Estructural de Contenidos y de Modelos Culturales” in: Suárez, H. (Ed), El Sentido y el Método. Sociología de la Cultura y Análisis de Contenido, El Colegio de Michoacán: Universidad Nacional Autónoma de México-Instituto de Investigaciones Sociales, Mexico, D. F., pp. $67-118$

Lefebvre, H. (1975), “Introducción al Estudio del Hábitat de Pabellón”, in Gaviria, M. (Ed), De lo Rural a lo Urbano, Ediciones Península, Barcelona, pp. 151-172.

Llano, F. (2014), "Espacios olvidados, lugares diferenciados: Transformación social del espacio urbano en Bogotá (1850-1880)”, Revista Nodo, Vol. 9, No 17, 2014, pp. 7-82.

Llano, F \& Chavarro, C (2013) pedagogía y ciudad: de las representaciones de la ciudad a la enseñanza de la ciudad. Revista Unipluriversidad Universidad de Antioquia

Llano, F. A (2013). Las facultades de educación y la formación docente en Colombia. En F.-A. Llano, A.-C. Osorio, J. Santamaría y R. Osorio. Entre lo deseable y lo posible. Historia de la facultad de ciencias de la educación 1960-2010. Bogotá: Ediciones Universidad La Gran Colombia.

López, A. (2016). “Dinámicas de la Arquitectura Colectiva” Entrevista personal.

Mazzanti, G. (2012,). "Model of the España Library", available at https://www.moma.org/collection/works/146715?locale=en (accessed 24 september 2017).

Orjuela, R., (2017) “Arquitectura Expandida " speak at UNAD University, Available at: https://www.youtube.com/watch?v=VA5AQqnu-jg (Accessed: 1 August 2018).

Pinzón, A,M. (2011) “La Autogestión Como Práctica De La comunidad y De Los Colectivos para Recuperar Espacios Comunitarios" Interview to Ana Lopez Available at http://www.a57.org/articulos/proyecto/Alaska-Parque-Comunal (Accessed: 1 August 2018). 
Rocha. J.L (2014) "Biblioteca España Sí... pero no así" Available at: http://narracciones.org/contenidos/biblioteca-espana-no-asi/ (accessed 31 July 2018) (Accessed: 1 August 2018).

Rodríguez, J,L "Debate sobre la Biblioteca España-segunda parte-publicado en esferapublica" http://www.torredebabel.info/debate-sobre-la-biblioteca-espana-segunda-parte-publicado-enesferapublica/ (accessed 31 July 2018)

Saldarriaga, A. (1996), Aprender Arquitectura, un Manual de Supervivencia, Corona, Bogotá.

Valencia, N. (2015) Ana López Ortego de Arquitectura Expandida: 'Me considero más activista social que arquitecta' | ArchDaily Colombia, Oct 30. Available at:https://www.archdaily.co/co/775871/analopez-ortego-de-arquitectura-expandida-me-considero-mas-activista-social-que-arquitecta (Accessed: 1 August 2018).

Wacquan, L. (2006), “Castigar a los Parias Urbanos” Antípoda Revista de Antropología y Arqueología, N², pp. 59-66

Waisman, M. (1985), "Critica del Concepto de Arquitectura y Ensayo de La Delimitación de su Nuevo Territorio" in ", La Estructura Histórica del Entorno, Ediciones Nueva Visión, Buenos Aires, Argentina, $p$

\section{Referencias secundarias}

Arasanz, A. (2010) "Medellín es Chevere". El País España, Nov 10 Available at: https://elviajero.elpais.com/elviajero/2010/11/10/actualidad/1289385245_850215.html (accessed 31 July 2018) 
Arquitectura Expandida (2014) "Do it Yourself" Available at: http://arquitecturaexpandida.org/category/procomun/ Accessed: 1 August 2018

Duque (2014), Políticas públicas, urbanismo y fronteras invisibles. Las disputas por el control espacial en Medellín xiii Coloquio Internacional de Geo crítica El control del espacio y los espacios de control Barcelona, 5-10 de mayo de 2014 